\title{
Intravascular ultrasound-guided emergency coronary Palmaz-Schatz stent placement without post-procedural systemic anticoagulation
}

\author{
Rudolf Blasini, Franz-Josef Neumann, Gert Richardt, Claus Schmitt, Ralf Paloncy, \\ Albert Schömig
}

\begin{abstract}
Objective-To test the efficacy of intravascular ultrasound (IVUS)-guided stent placement and to determine the clinical outcome during the first $\mathbf{3 0}$ days in those patients who were treated with antiplatelet therapy rather than anticoagulants because they met the IVUS criteria for optimal stent placement.

Design-Prospective observational study. Patients-126 patients with successful, non-elective Palmaz-Schatz stent placement.

Interventions-IVUS was performed to assess the attachment of stent struts, the coverage of the dissection, and the intrastent minimal lumen area.

Main outcome measures-Intrastent lumen area, clinical outcome during the first 30 days.

Results-In all patients IVUS showed complete apposition and coverage of the dissection. In 23 patients (18\%) the IVUS lumen area criterion was achieved. In 75 patients, further balloon dilatation was performed and in 41 IVUS criteria were finally fulfilled. The minimal intrastent lumen area increased from a mean (SD) of $6.81(1.15) \mathrm{mm}^{2}$ to $9.56(2.61) \mathrm{mm}^{2}(P \leqslant$ 0.01 ) between the first and final IVUS investigations. 64 patients $(51 \%)$ who met the IVUS criteria were treated with aspirin $(100 \mathrm{mg}$ ) and ticlopidine $(250 \mathrm{mg})$ twice a day. During the first 30 days none of the following events occurred: death, myocardial infarction, repeat intervention, aortocoronary bypass surgery, and subacute stent thrombosis.

Conclusion-The additional information provided by IVUS examination helped the operator to decide whether further dilatation was needed after a coronary stent had been placed. For patients who met the IVUS criteria for optimal stent placement, antiplatelet therapy was associated with an excellent clinical outcome during the first 30 days.
\end{abstract}

(Heart 1996;76:344-349)

Keywords: coronary stents; angioplasty; intravascular ultrasound; antiplatelet therapy

Coronary stenting is a reliable treatment for current or imminent vessel closure after balloon angioplasty (PTCA). ${ }^{1-3}$ Recent studies demonstrate that stent implantation after
PTCA of de novo stenoses reduces the restenosis rate. ${ }^{45}$ However, subacute stent thrombosis as well as bleeding and vascular complications caused by the intense anticoagulation are major limitations of coronary stent implantation..$^{6-8}$ Studies indicate that intravascular ultrasound imaging (IVUS) is better than angiography in the assessment of intrastent minimal lumen area. ${ }^{910}$ If an adequate lumen area is achieved by using IVUS, postprocedural anticoagulation may not be necessary. The initial clinical experience with patients receiving antiplatelet therapy after IVUS-guided coronary stent placement was encouraging. ${ }^{11-14}$

The purpose of the present study was to test the efficacy of IVUS-guided stent placement in avoiding postprocedural systemic anticoagulation. There were three primary objectives: firstly, to identify those patients who meet IVUS criteria for optimal stent placement; secondly, to assess the value of repeat dilatation in terms of the increase in lumen area; and thirdly, to determine the safety of antiplatelet therapy based upon IVUS criteria in terms of cardiac and non-cardiac clinical events during the first 30 days after stent placement.

\section{Patients and methods}

We studied 126 patients with symptomatic ischaemic heart disease who underwent successful intracoronary stent placement after balloon angioplasty (PTCA) at our hospital. Successful stent implantation was defined as stent placement at the desired position, a residual stenosis of less than $10 \%$, and a complete coverage of the dissection according to angiographic criteria. Indications for stent placement were coronary dissections after PTCA with progressive lumen narrowing within $10 \mathrm{~min}$, complete vessel closure, residual stenosis of $30 \%$ or more, and target lesions located in venous bypass grafts. According to previously published data, ${ }^{15}{ }^{16}$ marked irregularity of the vessel wall, a luminal filling defect suggestive of an intimal flap, or extravasation of contrast outside the lumen after dilatation were interpreted as angiographic evidence of coronary dissection. A progressive lumen narrowing with a residual stenosis of $>75 \%$ despite multiple balloon inflations disclosed by injection of contrast medium into the coronary artery was regarded as a threatened occlusion. Vessel closure was angiographically defined as TIMI flow grade 0 or $1 .{ }^{17}$ These definitions were applied to native vessels and also to venous bypasses. 
IVUS was performed in all patients in whom the stent had been successfully placed. The length of the dissection and the number of the segments implanted with a stent did not influence the decision to perform IVUS.

All patients were on long term treatment with low dose aspirin $(100 \mathrm{mg} / \mathrm{d})$, and their antianginal therapy was continued. During the procedure 15000 units of heparin and $500 \mathrm{mg}$ of aspirin was administered intravenously.

Before every angioplasty procedure, detailed information was given to all patients about stenting, the special management required, the IVUS investigation, and the inherent risks of the intervention. All patients gave written informed consent.

Stent implantation was performed according to a previously described technique using the femoral approach. ${ }^{15}{ }^{16} \mathrm{~A}$ conventional balloon catheter was used for angioplasty. An articulated $15 \mathrm{~mm}$ or $7 \mathrm{~mm}$ Palmaz-Schatz stent (Johnson \& Johnson, Warren, NJ) was hand-crimped on the angioplasty balloon. The balloon-stent assembly was advanced across the lesion site via a 0.014 inch guidewire which was left in place after PTCA. The size of the balloon catheter for stent deployment was chosen according to the angiographic estimation of the average proximal and distal reference segments with a tendency toward slight oversizing. The stent was positioned within the centre of the lesion with the aid of fluoroscopy. Balloon inflation with cine film documentation of complete balloon expansion was used to deploy the stent. After balloon deflation, the balloon catheter was removed and angiography was performed without foreshortening or vessel overlap. To achieve optimal stent expansion according to angiographic criteria, redilatation was carried out with the original balloon until an acceptable angiographic result was obtained.

In a next step, IVUS was performed with a Cardiovascular Imaging Systems (CVIS) system using $2 \cdot 9 \mathrm{~F}$ ultrasound catheters. The catheter has a mechanically rotating system with a $30 \mathrm{MHz}$ ultrasound crystal within the distal tip of a rotating wire. Using fluoroscopy, the imaging catheter is advanced via the guidewire until the distal marker crosses the distal reference segment by $20 \mathrm{~mm}$. The guidewire is then retracted under fluoroscopic control, and the imaging core is advanced. The ultrasound catheter was advanced to a site distal to the stented segment. The IVUS catheter was retracted manually. All IVUS studies were recorded on Super VHS videotape (Panasonic 7330E, Matsushita Electric) with an audio commentary on the videotape to describe the procedure. A cinefilm was recorded simultaneously to document the start and end of retraction. Special care was taken to document the distal and proximal reference diameters, the most distal and proximal ends of the stent, the bridge segment, and the tightest site.

\section{ON-LINE MEASUREMENTS}

The pullback of the catheter was reviewed to identify the tightest intra-stent site. Manual tracing of the lumen-intima border was performed by an experienced operator during the procedure to assess the lumen area of the tightest intra-stent site and of the distal and proximal reference segments (fig 1).

The following IVUS criteria defined optimal stent expansion:

- Complete apposition of the stent struts against the vessel wall.

- Complete coverage of the dissection.

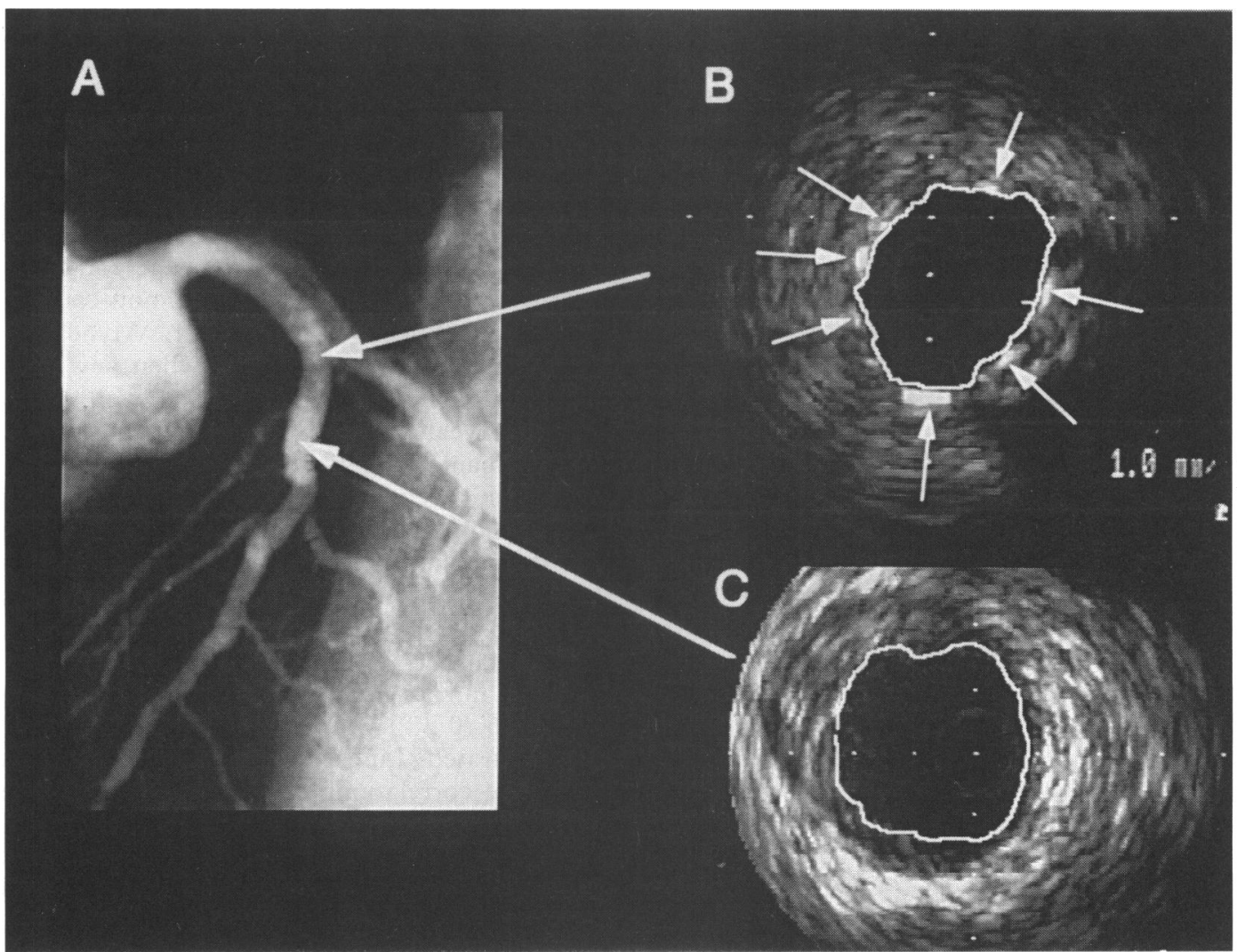

Figure 1 Example of an IVUS examination after stent implantation in the left anterior descending coronary artery. (A) angiography, (B) intrastent segment, $(C)$ distal reference segment; the luminal contour is shown in $B$ and $C$, arrows indicate the stent struts (B). 
- Intra-stent minimal lumen area $>8 \mathrm{~mm}^{2}$ and/or an intra-stent lumen area at the tightest site $>90 \%$ of the reference segment (an average of the proximal and distal values).

If the first IVUS evaluation showed that all criteria were met, the IVUS catheter was removed and a final angiography was performed. The procedure was then completed.

If the IVUS criteria were not met, further dilatations with larger balloons, high pressure balloons, and/or additional stent implantation were performed at operator's discretion to fulfil the IVUS criteria. The balloon used for repeat dilatation was chosen according to the results of the first IVUS examination, and matched in size the mean reference diameter. High pressure dilatation was applied when, despite the use of an appropriate balloon, adequate stent expansion could not be demonstrated. When a $7 \mathrm{~mm}$ long Palmaz-Schatz stent had been implanted short balloons were used for high-pressure dilatation.

These procedures were monitored by repeated IVUS evaluation. The intervention was complete when the IVUS criteria were met or when continuation of the interventional procedure would have increased the risk of complications.

\section{OFF-LINE ANALYSIS}

Consecutive IVUS images of optimal quality showing the ultrasound probe in a central and coaxial position were digitised into a $640 \times$ 480 dot matrix (media grabber, RasterOps Corporation) linked to an Apple Macintosh computer. All IVUS measurements were performed during diastole. The following measurements were then performed using standard analysis software (Image $1.40 \mathrm{NIH}$ ): minimal and maximal luminal diameters, lumen area. The validity of IVUS measurements was documented in vitro and in vivo. The reproducibility of IVUS dimension measurements was assessed by analysis of repetitive IVUS pullback sequences at three locations-that is, within the proximal and distal reference segments to stent transition as well as within the tightest portion of the stent. The mean (SD) difference between two measurements at 23 corresponding locations was $0.02(0.13) \mathrm{mm}$ for minimal lumen diameter and $0.04(0.33) \mathrm{mm}^{2}$ for cross sectional lumen area. Linear regression analysis revealed close correlations for minimal lumen diameter ( $r=$ $0 \cdot 88$ ) and cross sectional lumen area ( $r=$ $0 \cdot 97) .{ }^{9}$ The reproducibility of the off-line measurements was investigated by measuring the corresponding sites in the stent area and its reference area. By matching the values of the corresponding measurements recorded at 27 sites, a difference of $0.03(0.11) \mathrm{mm}$ in minimal lumen diameter and of $0.06(0.28) \mathrm{mm}^{2}$ in lumen area could be demonstrated. Linear regression analysis revealed close correlations for minimal lumen diameter $(r=0.89)$ and cross sectional lumen area $(r=0.93)$.

Quantitative coronary angiography was performed according to a previously described and validated edge detection algorithm based on the weighted sum of the first and second derivates of the digitised brightness information. ${ }^{17}$ The optimal view for documenting the stented lesion was selected for computer analysis from technically suitable angiograms. A $35 \mathrm{~mm}$ cinefilm was used at a speed of 25 frames per second. The catheter tip not filled with contrast was filmed near the centre of the screen and was taken as a reference.

\section{ANTICOAGULATION REGIMEN}

Patients fulfilling IVUS criteria were treated with heparin according to their activated partial thromboplastin time during the first 12 hours after stent placement and received aspirin $(100 \mathrm{mg})$ twice a day and ticlopidine $(250 \mathrm{mg})$ twice a day for the first 30 days after stent placement.

Patients not fulfilling the IVUS criteria were treated according to a previously described standard anticoagulation regimen. ${ }^{2}$ Heparin infusion was started immediately after the pressure bandage application and titrated to maintain the activated partial thromboplastin time $>80 \mathrm{~s}$. Immediately after the procedure, administration of an oral vitamin $K$ antagonist (Marcumar, Hoffmann-La Roche AG) and of aspirin (100 mg) twice a day were started. Heparin was continued until a stable level of oral anticoagulation was achieved (an international normalised ratio (INR) between 3.5 and 4.5). Oral anticoagulation was stopped four weeks after the procedure.

\section{CLINICAL EVENTS}

Between the stent implantation and in the next 30 days, death, myocardial infarction, repeat intervention (coronary artery bypass surgery and balloon angioplasty), subacute stent occlusion, bleeding complications, and vascular complications were noted. White cell and differential cell counts were monitored in the patients treated with ticlopidine. The 30-day follow up was completed in all patients enrolled in the study.

\section{DEFINITIONS}

The following definitions were used ${ }^{216}$ : all deaths were considered to be of cardiac origin unless a non-cardiac cause was detected at necropsy. Myocardial infarction was diagnosed when new pathological $Q$ waves developed that were not present on the baseline ECG or if there was an increase in creatine kinase to greater than twice the upper limit of normal or the creatine kinase myocardial isoenzyme was present, or both. Subacute stent occlusion was defined as angiographically confirmed occlusion of the stented vessel within the first 30 days after the intervention. Aortocoronary bypass surgery was defined as emergency bypass surgery involving the previously treated vessel. Repeat intervention was defined as PTCA in the previously treated vessel in association with angina pectoris, objective evidence of myocardial ischaemia, or both after the completion of the initial procedure. A bleeding complication was defined as requiring transfusion or surgery or as bleeding with associated organ dysfunction. Vascular com- 
Table 1 Clinical and angiographic characteristics of the 125 patients

\begin{tabular}{lc}
\hline Characteristic & Value $^{*}$ \\
\hline Age (y) & $59 \cdot 2(10 \cdot 5)$ \\
Women (\%) & $27(22)$ \\
Smokers (\%) & $52(42)$ \\
Hypercholesterolaemia (\%) & $47(38)$ \\
Arterial hypertension (\%) & $58(46)$ \\
Diabetes mellitus (\%) & $20(16)$ \\
Multivessel disease (\%) & $99(79)$ \\
Previous myocardial infarction (\%) & $42(43)$ \\
Acute myocardial infarction (\%) & $4(3)$ \\
Unstable angina pectoris (\%) & $54(43)$ \\
Exertional angina pectoris (\%) & $67(54)$ \\
Target vessel: & 125 \\
LAD (\%) & $50(40)$ \\
LCX (\%) & $22(18)$ \\
RCA (\%) & $48(38)$ \\
ACVB (\%) & $5(4)$ \\
Before stent implantation: & $3 \cdot 18(0 \cdot 49)$ \\
Reference diameter (mm) & $0 \cdot 73(0 \cdot 50)$ \\
Minimal lumen diameter (mm) & $78(17)$ \\
Stenosis \% of lumen diameter & $3 \cdot 23(0 \cdot 56)$ \\
After stent implantation: & $3 \cdot 02(0 \cdot 46)$ \\
Reference diameter (mm) & $7(2)$ \\
Minimal lumen diameter (mm) & $3 \cdot 25(0 \cdot 47)$ \\
Stenosis \% of lumen diameter & $1 \cdot 00(0 \cdot 07)$ \\
Measured balloon diameter (mm) & $12 \cdot 8(2 \cdot 2)$ \\
Balloon/artery ratio & $3 \cdot 45(1 \cdot 85)$ \\
Maximal balloon pressure (atm) &
\end{tabular}

$\mathrm{LAD}$, left anterior descending artery; LCX, left circumflex artery; RCA, right coronary artery; $\mathrm{ACVB}$, aorto-coronary venous bypass graft. ${ }^{\star}$ Data are expressed as mean (SD) or as numACVB, a
bers (\%).

plications were aneurysm, pseudoaneurysm, or arteriovenous fistula requiring surgery. Cerebrovascular or other non-cardiac nonbleeding events were recorded as well. In addition, any haematological side effects of the antiplatelet therapy were noted. The patients were observed for 10 to 14 days in the hospital. Clinical outcome was assessed by regular follow up in the outpatient clinic, physical examination, and ECG during the four to six weeks after stent placement.

\section{STATISTICAL ANALYSIS}

Values were reported as mean (SD). Statistical analyses were performed with Stat View software (Abacus Concepts, Berkeley, CA). Intraindividual comparisons were made using the paired $t$ test. A difference was regarded as significant when the two-sided test value was $\mathrm{P}<$ 0.05 .

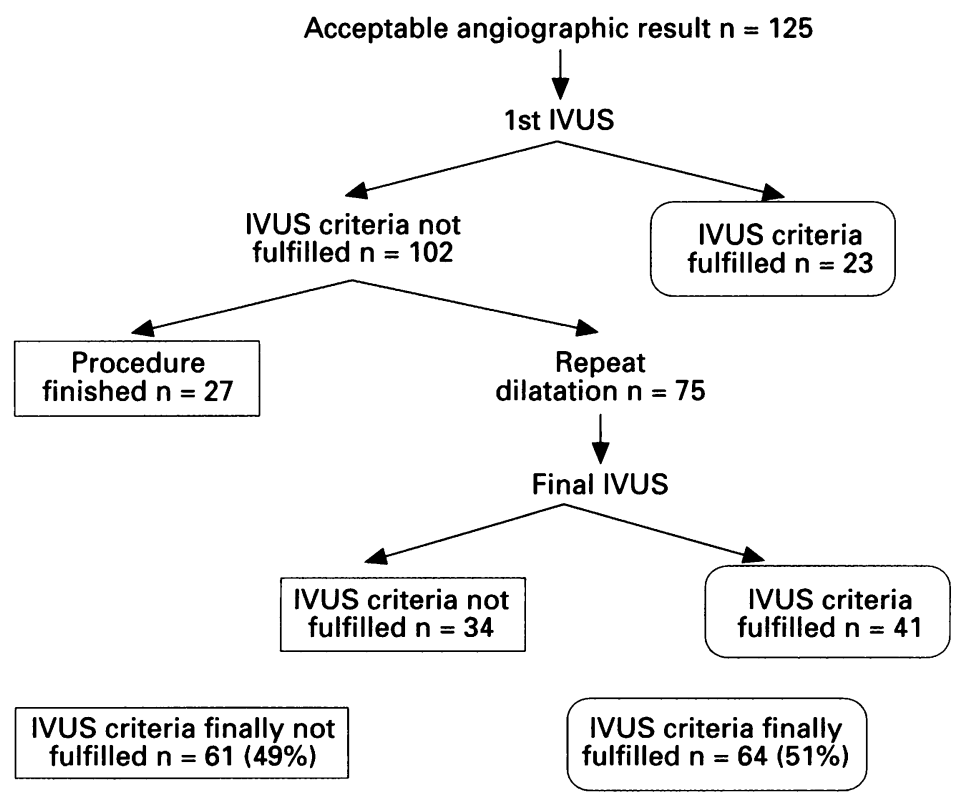

Figure 2 Flow diagram showing the procedure after an acceptable angiographic result was obtained.

\section{Results}

Coronary stenting guided by IVUS was planned for 126 patients and the ultrasound imaging catheter was successfully placed in 125 patients $(99 \%)$. In one patient, the imaging core could not be advanced.

Table 1 shows the baseline clinical and angiographic characteristics of our patients. After stent implantation, the angiographically assessed minimal lumen diameter was 3.02 $(0.46) \mathrm{mm}$, the reference diameter was 3.23 $(0.56)$, and a residual diameter stenosis of 7 (2)\% was calculated. The balloon size of the last balloon before IVUS investigation was $3.25(0.47) \mathrm{mm}$ and the maximal inflation pressure was $12 \cdot 8(2 \cdot 2)$ atm.

\section{FIRST IVUS EXAMINATION}

In all patients there was complete apposition of the stent struts against the vessel wall and complete coverage of the dissection was documented. The IVUS lumen area criterion was met in 23 patients (18\%) (fig 2). In these patients, the minimal stent area was 8.62 $(1 \cdot 17) \mathrm{mm}^{2}$ and the procedure was completed after the first IVUS examination. A total of 102 patients did not meet the IVUS criteria. In 27 patients, additional dilatation was not performed, because the operator considered further attempts a major risk. In these patients the lumen area was $6.32(1.76) \mathrm{mm}^{2}$.

In 75 patients repeat balloon inflations with larger balloons were performed. The size of the final balloon was $3.72(0.49) \mathrm{mm}$ and the maximal inflation pressure was $13.0(1 \cdot 7) \mathrm{atm}$.

\section{FINAL IVUS EXAMINATION}

In another 41 patients IVUS criteria were finally met after repeat dilatation. These patients showed an improvement in minimal stent area from $6.81(1.15) \mathrm{mm}^{2}$ (first IVUS) to $9.56(2.61) \mathrm{mm}^{2}(\mathrm{P}<0.01)$ (fig 3, table 2$)$.

In 34 patients the IVUS lumen area criterion was not fulfilled despite further dilatations. The minimal stent area was enlarged from $5.94(1.31) \mathrm{mm}^{2}$ to $6.62(1.54) \mathrm{mm}^{2}$ $(\mathrm{P}<0.05)$.

SIDE EFFECTS OF IVUS INVESTIGATION Three patients displayed vasospasm distal to

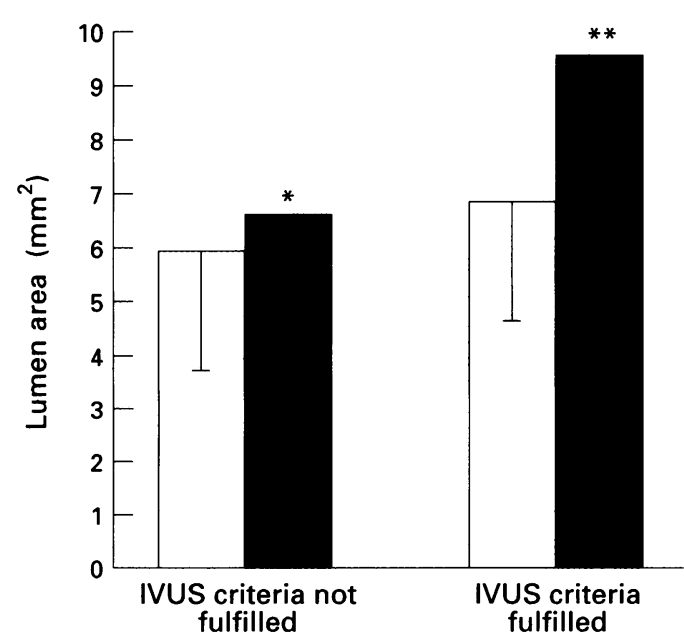

Figure 3 Minimal intra-stent lumen area before (open bars) and after (solid bars) further dilatation. 
Table 2 Intravascular ultrasound data (mean (SD))

\begin{tabular}{|c|c|c|}
\hline Variable & $\begin{array}{l}\text { IVUS criteria } \\
\text { finally fulfilled }\end{array}$ & $\begin{array}{l}\text { IVUS criteria } \\
\text { finally not fulfilled }\end{array}$ \\
\hline $\begin{array}{l}\text { Reference lumen area }\left(\mathrm{mm}^{2}\right) \\
\text { Minimal lumen area } 1 \mathrm{st} \text { IVUS }\left(\mathrm{mm}^{2}\right) \\
\text { Minimal lumen area final IVUS }\left(\mathrm{mm}^{2}\right) \\
\text { Reference minimal diameter (IVUS) (mm) } \\
\text { Reference minimal diameter (QCA) (mm) } \\
\text { Minimal diameter lst IVUS (IVUS) (mm) } \\
\text { Minimal diameter 1st IVUS (QCA) (mm) } \\
\text { Minimal diameter final IVUS (IVUS) (mm) } \\
\text { Minimal diameter final IVUS (QCA) (mm) }\end{array}$ & $\begin{array}{r}10 \cdot 02(2 \cdot 50) \\
6 \cdot 81(1 \cdot 15) \\
9 \cdot 56(2 \cdot 61) \\
3 \cdot 10(0.44) \\
3.37(0.61) \\
2 \cdot 19(0 \cdot 30) \\
2 \cdot 88(0.43) \\
2 \cdot 81(0.37) \\
3 \cdot 11(0.46)\end{array}$ & $\begin{array}{l}8.71(3.18) \\
5.94(1.31) \\
6.62(1.54) \\
3.05(0.52) \\
3.07(0.46) \\
2.20(0.28) \\
2.69(0.41) \\
2.57(0.36) \\
3.07(0.45)\end{array}$ \\
\hline
\end{tabular}

IVUS, intravascular ultrasound; QCA, quantitative coronary angiography.

the stented segment after IVUS investigation. The spasm was fully reversible after administration of intracoronary glyceryl trinitrate. No further side effects were noted.

\section{CLINICAL OUTCOME}

According to the predefined IVUS criteria, 64 patients were allocated to aspirin-ticlopidine treatment. In this group of patients none of the following events occurred during the first 30 days after stent implantation: death, myocardial infarction, repeat intervention, aortocoronary bypass surgery, and subacute stent occlusion. One patient bled from a gastric ulcer. There were no vascular complications in any of the patients. No haematological disorder was seen in any of our patients who were treated with aspirin and ticlopidine for 4 weeks.

\section{ANGIOGRAPHIC FOLLOW UP}

A second coronary angiography was performed after six months in $74 \%$ of the eligible patients in whom IVUS optimisation had been planned. This showed a restenosis rate with a minimal intrastent diameter of less than $50 \%$ in $20.9 \%$ of the patients.

\section{Discussion}

This prospective study of patients who underwent non-elective coronary stent implantation demonstrates that IVUS identifies many patients with suboptimal stent deployment despite an angiographically acceptable result. Furthermore, the IVUS data indicate that additional dilatation is associated with a significant increase in lumen area. In patients who met predefined IVUS criteria, antiplatelet therapy with aspirin and ticlopidine was not associated either with cardiac events or with vascular and bleeding complications during the first 30 days after stent placement.

\section{IVUS FOR THE ASSESSMENT OF THE STENT} RESULT

Coronary stents challenge imaging techniques because the thin Palmaz- Schatz stent struts are nearly radiolucent. Tomographic imaging methods seem an appropriate way of assessing the interaction of the stent and vessel wall and intra-stent lumen area..$^{9118}$ In earlier studies IVUS was better than angiography for determining the minimal intra-stent lumen area..$^{20-22}$ Therefore, the primary objectives of the present study were to identify those patients who do not meet predefined criteria for optimal stent placement and secondly to evaluate the success of repeat dilatation prompted by IVUS data. The findings of our study confirm IVUS as an important diagnostic tool: despite an angiographically acceptable result only 23 out of 125 patients complied with the predefined IVUS criteria. Further dilatation with larger balloons was performed in 75 patients, leading to a significant increase in the intra-stent minimal lumen area of $40 \%$. We used the lumen area of the reference segment instead of the vessel area (that is, the area within the lamina elastica externa) for balloon sizing. ${ }^{1422}$ Since the vessel area depends highly on the extent to which a vessel is enlarged to compensate for plaque, the lumen area seems to be the appropriate criterion for the adjustment of the balloon size. When this index was applied to repeat dilatation, 64 out of 125 patients complied with the predefined criteria.

\section{ANTIPLATELET THERAPY AFTER CORONARY STENT PLACEMENT}

If an adequate intra-stent lumen area is achieved after coronary stent placement anticoagulation may be unnecessary. Residual intrastent stenosis is assumed to be associated with an increased risk of stent thrombosis. ${ }^{2324}$ Therefore, stent placement without post-procedural anticoagulation is considered possible in patients with IVUS-based optimised repeat dilatation. These patients display a very low rate of adverse clinical events during the first 30 days after stent placement. In our study group of 64 patients, no stent occlusion, myocardial infarction, and repeat intervention occurred. There were no vascular complications and only one patient suffered a bleeding complication; this was caused by a gastric ulcer. These results accord with the data previously published for patients with elective coronary stent placement ${ }^{14}$ which showed a much lower event rate than that in patients treated by the standard anticoagulation regimen. ${ }^{267}$

\section{STUDY LIMITATIONS}

The study was designed to evaluate the feasibility of using IVUS and its efficacy in the assessment of the result of stent placement and the success of repeat dilatation based on IVUS criteria. Accordingly, we did not randomise or compare our patients with a control group not receiving IVUS.

The design of the study does not allow for a comparison of the clinical outcome of the patients receiving antiplatelet and those receiving anticoagulation therapy because the two groups were quite heterogenous, as the study protocol shows. Moreover, we did not randomise the medical regimen, and thus, no control group was set up. Consequently, this does not allow us to draw significant conclusions regarding the need for IVUS during stent implantation.

The IVUS criteria were arbitrarily chosen. An absolute lumen area of $8.0 \mathrm{~mm}^{2}$ and/or a ratio of 0.9 between minimal stent cross sectional area and average cross sectional lumen area of the reference segments was considered adequate. The indication for and the performance of further stent dilatation was based on 
an anticipated elastic recoil of the PalmazSchatz stent of about $15 \%$, on a balloon size which was determined according to the reference diameters, and on a balloon : vessel ratio of $1 \cdot 0: 1 \cdot 1 .^{15}$ These criteria assume that there is no major risk of severe vessel injury associated with the intra-stent dilatation.

Because of the high risk involved in further dilatation the operator decided to end the intervention before IVUS criteria were fully met in 24 patients after the first IVUS examination and in 34 patients after a repeat dilatation with larger balloons. It remains unclear whether the intense use of short, non-compliant high-pressure balloons for repeat dilatation would have achieved further luminal gain in these patients. ${ }^{14}$ Furthermore, we cannot rule out the possibility that many patients would initially have met the IVUS criteria if the first dilatation had been performed with a highpressure balloon.

This study was not designed to determine whether IVUS is a prerequisite for the indication for treatment with antiplatelet medication. Randomised trials are needed to answer this question. For this reason, we are conducting a study in which, without previous IVUS examination after stent implantation, antiplatelet therapy with aspirin and ticlopidine is tested against conventional treatment by heparin, vitamin $K$ antagonist, and aspirin. ${ }^{25} \mathrm{~A}$ study that investigates the importance of high-pressure dilatation with respect to the rate of subacute complications and the restenosis rate has yet to be performed.

A potential limitation of our investigation is that the pullback of the IVUS catheter was carried out manually and not by a motor. However, when pulling back the catheter, the investigators took great care to identify and determine the section of the smallest stent area by conducting numerous measurements.

Our study did not examine whether aspirin or ticlopidine alone or in combination can be regarded as optimal antiplatelet therapy. This question was addressed in a recent study. ${ }^{11}$

Ticlopidine is a novel platelet antiaggregant that functions primarily as an inhibitor of the adenosine diphosphate pathway of platelet aggregation. ${ }^{19}$ Thus a synergistic effect of aspirin and ticlopidine may be assumed because aspirin inhibits the cyclooxygenase pathway.

\section{CONCLUSIONS}

We found that IVUS examination was a valuable diagnostic technique that helped us to decide whether further dilatation was necessary after coronary stent placement. We showed that though the angiographic result may be acceptable, a far more significant acute increase in lumen area can be achieved by IVUS-based repeat dilatation. Furthermore, we concluded that postprocedural oral anticoagulation was unnecessary, at least for patients who met the IVUS criteria, because there were no cardiac complications and hardly any bleeding or vascular complications during the first 30 days of antiplatelet therapy with aspirin-ticlopidine treatment.
1 George BS, Voorhees W, Roubin GS, Fearnot NE, Pinkerton CA, Raizner AE, et al. Multicenter investiPinkerton CA, Raizner AE, et al. Multicenter investigation of coronary stenting to treat acute or threatened closure after percutaneous transluminal coronary angioplasty: clinical and ang

2 Schömig A, Kastrati A, Mudra H, Blasini R, Schuhlen H, Klauss V, et al. Four-year experience with Palmaz-Schatz stenting in coronary angioplasty complicated by dissection with threatened or present vessel closure. Circulation 1994;90:2716-24.

3 Roubin GS, Cannon AD, Agrawal SK, Macander PJ, Dean LS, Baxley WA, et al. Intracoronary stenting for acute and threatened closure complicating percutaneous transluminal coronary angioplasty. Circulation 1992;85: 916-27.

4 Fischman DL, Leon MB, Baim DS, Schatz RA, Savage MP, Penn I, et al. A randomized comparison of coronary-stent placement and balloon angioplasty in the treatment of coronary artery disease. Stent Restenosis Study Investigators. N Engl f Med 1994;331:496-501.

5 Serruys PW, de Jaegere P, Kiemeneij F, Macaya C, Rutsch W, Heyndrickx G, et al. A comparison of balloonexpandable-stent implantation with balloon angioplasty in patients with coronary artery disease. Benestent Study Group. N Engl F Med 1994;331:489-95.

6 Schatz RA, Goldberg S, Leon M, Baim D, Hirshfeld J, Cleman $M$, et al. Clinical experience with the PalmazSchatz coronary stent. f Am Coll Cardiol 1991;17: $155-9$.

7 Schatz RA, Baim DS, Leon M, Ellis SG, Goldberg S, Hirshfeld JW, et al. Clinical experience with the PalmazSchatz coronary stent. Initial results of a multicenter study. Circulation 1991;83:148-61.

8 study. Circulation 1991;83:148-61. Chapekis A, Quain L, et al. Thrombosis of a flexible coil Chapekis A, Quain $\mathrm{L}$, et al. Thrombosis of a flexible coil coronary stent: frequency, predictors and

9 Mudra H, Klauss V, Blasini R, Kroetz M, Rieber J, Regar E, et al. Ultrasound guidance of Palmaz-Schatz intracoronary stenting with a combined intravascular ultrasound balloon catheter. Circulation 1994;90:1252-61.

10 Nakamura S, Colombo A, Gaglione A, Almagor $\mathrm{Y}$ Goldberg SL, Maiello L, et al. Intracoronary ultrasound observations during stent implantation. Circulation 1994;89:2026-34

11 Hall P, Nakamura S, Maiello L, Itoh A, Blengio S, Martini $\mathrm{G}$, et al. A randomized comparison of combined Ticlopidine and Aspirin therapy versus Aspirin therapy alone after successful intravascular ultrasound-guided stent implantation. Circuation 1996;93:215-22.

12 Russo RJ, Schatz RA, Morris NB, Stevens KM, Teirstein PS. Ultrasound guided coronary stent placement without Warfarin anticoagulation. Six-month clinical follow up. Circulation 1995;92:543.

13 Serruys PW, Di Mario C. Who is thrombogenic: the stent or the doctor? Circulation 1995;91:1891-3.

14 Colombo A, Hall P, Nakamura S, Almagor Y, Maiello L, Martini G, et al. Intracoronary stenting without anticoagulation accomplished with intravascular ultrasound. ulation accomplished with
Circulation 1995;91:1676-88.

15 Schömig A, Kastrati A, Dietz R, Rauch B, Neumann FJ, Katus HH, et al. Emergency coronary stenting for dissecKitus HH, et al. Emergency coronary stenting for dissecplasty: angiographic follow-up after stenting and after plasty: angiographic follow-up after stenting and after
repeat angioplasty of the stented segment. $f \mathrm{Am}$ Coll repeat angioplasty of the

16 Kastrati A, Schömig A, Dietz R, Neumann FJ, Richardt G. Time course of restenosis during the first year after emergency coronary stenting. Circulation 1993;87: 498-505.

17 Cheseboro JH, Knatterud G, Roberts R, Borer J, Cohen LS, Dalen J. Thrombolysis in myocardial infarction (TIMI) trial. Circulation 1987;76:142-54

18 Nishimura RA, Edwards WD, Warnes CA, Reeder GS, Holmes DJ, Tajik AJ, et al. Intravascular ultrasound imaging: in vitro validation and pathologic correlation. $\mathcal{F}$ Am Coll Cardiol 1990;16:145-54.

19 Hass WK, Easton JD, Adams HP Jr, Phillips W, Molony $\mathrm{BA}$, Anderson S, et al. A randomized trial comparing ticlopidine hydrochloride with aspirin for the prevention of stroke in high-risk patients. Ticlopidine Aspirin Stroke Study Group. N Engl 7 Med 1989;321:501-7.

20 Mudra H, Blasini R, Regar E, Klauss V, Rieber J, Theisen $\mathrm{K}$. Intravascular ultrasound assessment of the balloonexpandable Palmaz-Schatz coronary stent. Coron Artery Dis 1993;4:791-9.

21 Blasini R, Mudra H, Regar E, Neumann FJ, Kastrati A, Schömig A. Variable expansion pattern of intracoronary Palmaz Schatz stents: insights with intravascula ultrasound imaging (abstract). Eur Heart 7 1993;14:351.

22 Goldberg SL Colombo A Nakamura S, Almagor Y Maiello L, Tobis JM. Benefit of intracoronary ultrasound in the deployment of Palmaz-Schatz stents. $\mathcal{f} \mathrm{Am}$ Coll in the deployment of Palm.

23 Haude M, Erbel R, Issa H, Straub U, Rupprecht HJ, Treese $\mathrm{N}$, et al. Subacute thrombotic complications after Heart $f$ 1993; 126:15-22.

24 Noldge G, Richter GM, Siegerstetter V, Garcia O, Palmaz JC. Tierexperimentelle Untersuchungen uber den Einfluss der Flussrestriktion auf die Thrombogenitat des Palmaz-Stentes mittels 111 In

25 Schömig A, Neumann FJ, Kastrati A, Schühlen $\mathrm{H}$, Blasin $\mathrm{R}$, Hadamitzky $\mathrm{M}$, et al. A randomized comparison of $\mathrm{R}$, Hadamitzky $\mathrm{M}$, et al. A randomized comparison of stent placement. $N$ Engl भ Med 1996;334:1084-7. 